\title{
Editorial: Evolution of Mitochondrial Genomes
}

\author{
Sophie Breton ${ }^{1 *}$, Eric Pante ${ }^{2}$, Xiao-Feng Xue ${ }^{3}$ and Jing-Tao Sun ${ }^{3}$ \\ ${ }^{1}$ Department of Biological Sciences, Université de Montréal, Montréal, QC, Canada, ${ }^{2}$ Littoral Environnement et Sociétés \\ (LIENSS), UMR 7266, CNRS-La Rochelle Université, La Rochelle, France, ${ }^{3}$ Department of Entomology, Nanjing Agricultural \\ University, Nanjing, China
}

Keywords: mitochondrial DNA, evolutionary biology, phylogenetics, population genetics, mitogenomic comparison

\section{Editorial on the Research Topic}

\section{Evolution of Mitochondrial Genomes}

Animal mitochondria have their own genome (mtDNA), which is typically described as small $(\sim 16 \mathrm{~kb})$, circular and maternally inherited with 37 genes, i.e., 13 for protein-coding genes involved in ATP production, and 22 for transfer RNAs and two for ribosomal RNAs. However, recent data show that the functional repertoire of the animal mtDNA has been underestimated, even in humans. Moreover, while several animal groups are relatively conservative in terms of their mt genome content and organization, recent studies revealed that some groups exhibit an astonishing diversity in $\mathrm{mt}$ chromosome form and number as well as coding content and organization.

One aim of this Research Topic was to better understand the evolutionary processes that underlie mt genome diversity in animals and also other organisms (e.g., Nishimura et al.; Smith). For example, one major difference in mitochondrial gene content between animals and plants + some unicellular eukaryotes resides in the occurrence in the latter two of ccm genes (for cytochrome c maturation). In their study, Nishimura et al. were interested in two evolutionarily distinct systems for cytochrome $c$ maturation in mitochondria-Systems I and III- the former involves a set of mitochondrion-encoded proteins whereas the latter is operated exclusively by nucleus-encoded proteins. Specifically, the authors tested the previous hypothesis that cytochrome $c$ maturation switched from System I to III in the early evolution of Cryptista, one of the major taxonomic assemblages in eukaryotes, by sequencing two new mtDNAs from two key cryptist members. The two newly sequenced mtDNAs revealed a patchy distribution of the two evolutionarily distinct systems, and prompted the authors to revise previously proposed scenarios for the evolution of cytochrome c maturation in this group.

Another aim of this Research Topic was to disentangle the phylogeny and evolution of some animal groups at a wide scale, using characters of $\mathrm{mt}$ genomes or complete mtDNA sequences. Indeed, some characters, such as atypical secondary structures of transfer RNAs, which have been consistently found in nematodes, insects, spiders, mites and ticks, have potential value to decipher the phylogeny and evolution of animals at a higher level (class, order or family). For example, studies in insects (e.g., Du et al.) and nematodes (reviewed in Kern et al.) have shown that complete mtDNA sequences have higher resolutions than partial gene sequences for population genetics and phylogenetic studies, although combining several mitochondrial and nuclear genes also remain an appropriate approach. Overall, this Research Topic aimed in elucidating the genetic, life history, and ecological factors that contribute to the evolutionary dynamics of mt genomes in animals and other organisms. 


\section{AUTHOR CONTRIBUTIONS}

SB, EP, X-FX, and J-TS wrote the editorial. All authors contributed to the article and approved the submitted version.

Conflict of Interest: The authors declare that the research was conducted in the absence of any commercial or financial relationships that could be construed as a potential conflict of interest.
Copyright (c) 2020 Breton, Pante, Xue and Sun. This is an open-access article distributed under the terms of the Creative Commons Attribution License (CC BY). The use, distribution or reproduction in other forums is permitted, provided the original author(s) and the copyright owner(s) are credited and that the original publication in this journal is cited, in accordance with accepted academic practice. No use, distribution or reproduction is permitted which does not comply with these terms. 